\title{
Comparison of Radiologic Outcomes of Different Methods in Single-Level Anterior Cervical Discectomy and Fusion
}

\author{
O Ik Kwon, Dong Wuk Son, Sang Weon Lee, Geun Sung Song \\ Department of Neurosurgery, Pusan National University Yangsan Hospital, \\ Pusan National University School of Medicine, Yangsan, Korea
}

\begin{abstract}
Objective: Anterior cervical discectomy and fusion (ACDF) is a choice of surgical procedure for cervical degenerative diseases associated with radiculopathy or myelopathy. However, the patients undergoing ACDF still have problems. The purpose of the present study is to evaluate the radiologic results of 3 different methods in single-level ACDF.

Methods: We conducted a retrospective collection of radiological data from January 2011 to December 2014. A total of 67 patients were included in this study. The patients were divided into 3 groups by operation procedure: using stand-alone cage (group cage, $n=20$ ); polyether-ether-ketone (PEEK)-titanium combined anchored cage (group $A C, n=21$ ); and anterior cervical cage-plate (group $\mathrm{CP}, \mathrm{n}=26$ ). Global cervical lordosis ( $\mathrm{C} 2-\mathrm{C} 7 \mathrm{Cobb}$ angle), fused segment height, fusion rate, and cervical range of motion (ROM) were measured and analyzed at serial preoperative, postoperative, 6-month, and final 1-year follow-up.

Results: Successful bone fusion was achieved in all patients at the final follow-up examination; however, the loss of disc height over $3 \mathrm{~mm}$ at the surgical level was observed in 6 patients in group cage. Groups AC and CP yielded significantly better outcomes than group cage in fused segment height and cervical $\operatorname{ROM}(p=0.01$ and $p=0.02$, respectively). Furthermore, group AC had similar radiologic outcomes to those of group $\mathrm{CP}$.

Conclusion: The PEEK-titanium combined anchored cage may be a good alternative procedure in terms of reducing complications induced by plate after ACDF.
\end{abstract}

Key Words: Cervical vertebrae $\cdot$ Radiography $\cdot$ Postoperative complications $\cdot$ Spinal fusion

\section{INTRODUCTION}

Anterior cervical discectomy and fusion (ACDF) is a choice of surgical procedure for cervical degenerative diseases associated with radiculopathy or myelopathy ${ }^{12)}$. Since its first introduction by Smith and Robinson in $1958^{22}$, there have been many advances in ACDF for cervical degenerative diseases. Traditionally, iliac bone graft was used. However, several complications have been observed, including donor site hematoma, infection, and pain $^{21,27}$. To avoid these complications, appropriate techniques have been modified by many surgeons and manufacturers and the quality of medical instruments has been improved. The use of stand-alone cages has been suggested by some surgeons to avoid iliac bone graft related complica-

- Received: March 10, 2016 • Revised: July 6, 2016

- Accepted: August 1, 2016

Corresponding Author: Dong Wuk Son

Department of Neurosurgery, Pusan National University Yangsan

Hospital, Pusan National University School of Medicine, 20

Geumo-ro, Mulgeum-eup, Yangsan 50612, Korea

Tel: +82-55-360-2126, Fax: +82-55-360-2156

E-mail: oik99@naver.com

@This is an open access article distributed under the terms of the Creative Commons Attribution Non-Commercial License (http://creativecommons.org/licenses/by-nc/4.0/) which permits unrestricted non-commercial use, distribution, and reproduction in any medium, provided the original work is properly cited. tions. However, stand-alone cages have also been reported to have some complications, such as cage dislocation, subsidence, and cervical kyphosis ${ }^{1,2)}$. Therefore, the anterior cervical cageplate method has been widely used to provide stability and reduce complications related to the use of stand-alone cage ${ }^{5,6,16}$. However, the application of anterior cervical plate also has some complications, such as increasing incidence of dysphagia, esophageal injuries, and plate malposition ${ }^{3,7,17}$. For the purpose of diminishing potential complications of anterior cervical plate, a newly designed polyether-ether-ketone (PEEK)-titanium combined anchored cage was invented and applied for ACDF. However, the patients undergoing ACDF still have been concerned about complications after ACDF. Many studies have attempted to investigate the results after undergoing ACDF. However, few studies have undertaken a radiologic comparison of three ACDF methods: stand-alone cage, PEEK-titanium combined anchored cage, and anterior cervical cage-plate. The purpose of the present study is to evaluate the radiologic outcomes of these three different in single-level ACDF.

\section{MATERIALS AND METHODS}

\section{Patient Population}

We conducted a retrospective collection of radiological data in the patients with one-level cervical degenerative diseases 
who had undergone anterior cervical fusion at Pusan National University Yangsan Hospital from January 2011 to December 2014. The patients were only included in the sample when the following inclusion criteria were fulfilled: (1) no history of previous cervical spinal surgery; (2) indication for ACDF for single-level cervical disease; and (3) absence of concomitant spinal disease; spinal tumor, infection, fracture or subluxation. A total 67 patients were included in this study; 30 patients in cervical spondylotic radiculopathy, 22 patients in cervical spondylotic myelopathy, 15 patients in herniated intervertebral disc. The patients were divided into 3 groups by operation procedure: (1) using stand-alone cage (group cage, $\mathrm{n}=20$ ); (2) PEEK-titanium combined anchored cage (group AC, $n=21$ ); and (3) anterior cervical cage-plate (group $\mathrm{CP}, \mathrm{n}=26$ ). Serial preoperative, postoperative, 6-month, and final 1-year followup simple X-rays and computed tomography scans were evaluated for radiological evaluation. Global cervical lordosis (C2$\mathrm{C} 7 \mathrm{Cobb}$ angle), fused segment height (FSH), fusion rate and cervical range of motion (ROM) were measured and analyzed.

\section{Surgical Technique}

All the surgeries were performed by a chief surgeon of our hospital. All patients were operated with the standard antero-lateral approach. To define the operated level in supine position, the fluoroscope was used. After confirmation of operation levels, a portion of anterior longitudinal ligament and disc material were appropriately removed with a pneumatic drill and a Kerrison punch. During the discectomy using the microscope, while the cartilage endplates were removed with the curettage, we paid attention to avoid any additional damage to the endplate. A high-speed pneumatic drill and a Kerrison punch were used to decompress nerve roots by removing osteophytic overgrowth in the both uncovertebral joint, even in patients with unilateral symptoms. We limited uncovertebral resection within medial one-third to prevent instability. After decompression was complete, we used fluoroscopy for antero-posterior and lateral view in order to determine the cage size, the trajectory of screw, and the angle of the plate. As a Caspar distractor (Aesculap, Burlingame, CA, USA) was placed between adjacent vertebral bodies, the surgeon clicked a retractor to perform distraction approximately 2 to $3 \mathrm{~mm}$ until a point of excessive resistance. Trial spacers were used to determine the appropriate implant shape and size; a suitable PEEK (Cornerstone PSR, Medtronic Sofamor Danek USA Inc., Memphis, TN, USA) cage was inserted in group cage, the corresponding zero-profile anchored spacer (Spine, Depuy Synthes Co., Zuchwil, Switzerland) was placed and 4 screws were inserted under fluoroscopy in group AC. In group CP, an allograft tissue cage (Cornerstone ASR, Medtronic Sofamor Danek, Memphis, TN, USA) with anterior plate (Zephir, Medtronic Sofamor Danek) was placed safely under fluoroscopy. Anterior border of the cage was aligned with anterior rim of the vertebral body to prevent subsidence. After implantation of the cage into anterior margin of the disc space using an impactor, a Caspar distractor was released and the stability of the cage was confirmed by manual pullout test. All patients were instructed to wear a soft collar for 2 months after surgery.

\section{Radiological Assessment}

All of radiological assessments were performed by 2 independent observers highly experienced in spinal diseases. Plain anteroposterior and lateral radiographs were routinely obtained before operation and on the first postoperative day. These films were also taken at 6 months and 1 year after surgery. For the evaluation of the sagittal alignment of the cervical spine, the lateral radiographs of the cervical spine at the neutral position were measured using the Cobb angle. The Cobb angle is defined as the angle between a line drawn parallel to the inferior endplate of C2 vertebral body and C7 vertebral body. To evaluate $\mathrm{FSH}$, the mean values of the anterior and posterior vertebral body heights of the surgical level were measured (Fig. 1). Subsidence was identified as the sinking of the cage into vertebral body, the loss of disc height at the surgical level over $3 \mathrm{~mm}^{24)}$. Cervical ROM was measured as the difference of Cobb angle values between the extension and flexion positions.

\section{Statistical Analysis}

All results are represented as mean \pm standard deviation. Statistical analyses were performed using IBM SPSS Statistics ver. 21.0 (IBM Co., Armonk, NY, USA). Intergroup results were compared using analysis of variance, Kruskal-Wallis rank sum test, and Mann-Whitney U-test. Values of $\mathrm{p}<0.05$ were considered to be statistically significant.

\section{RESULTS}

\section{Patient Characteristics}

Following exclusion of the patients without the value of
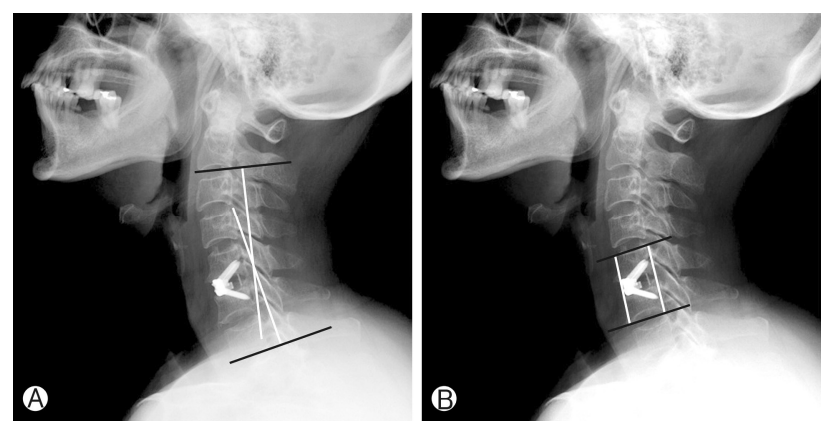

Fig. 1. Postoperative lateral rediographs showing (A) Cobb angle between $\mathrm{C} 2$ and $\mathrm{C} 7$ inferior endplate to estimate Global cervical lordosis. (B) Fused segment height, the mean value of anterior and posterior vertebral body heights at surgical level. 
bone marrow density (BMD) or adequate radiological images, a total of 67 patients were divided into 3 groups by operation procedure: (1) using stand-alone cage (group cage, $n=20$ ); (2) PEEK-titanium combined anchored cage (group AC, $n=21$ ); and (3) anterior cervical cage-plate (group $\mathrm{CP}, \mathrm{n}=26$ ). No significant differences in age, gender, and $\mathrm{BMD}$ were observed. The preoperative characteristics of the 3 groups are summarized in Table 1.

\section{Radiological Outcome}

Successful bone fusion was achieved in all patients at the final follow-up examination. However, loss of disc height over $3 \mathrm{~mm}$ at the surgical level was observed in 6 patients in group cage. To evaluate FSH change of the surgical level, the immediate postoperative height set to $100 \%$. While the loss of height occurred at 6 month after surgery in all groups, no statistically significant differences were observed for each group $(p>0.05)$. However, at 1-year follow-up, FSH decreased significantly as compared to 6-month postoperative image in all groups: $93.64 \% \pm 3.39 \%, 95.79 \% \pm 2.77 \%$, and $97.0 \% \pm 2.03 \%$ in groups cage, AC, and $\mathrm{CP}(\mathrm{p}=0.01)$ (Fig. 2). The results of a multiple comparison analysis showed a statistically significant decrease of FSH in group cage as compared to group $\mathrm{CP}(\mathrm{p}=0.01)$. The comparison between groups $\mathrm{AC}$ and $\mathrm{CP}$ showed no significant differences $(\mathrm{p}>0.05)$. The $\mathrm{C} 2-\mathrm{C} 7 \mathrm{Cobb}$ angle immediately after the surgery was $14.76 \pm 4.62$ for group cage, $12.26 \pm 6.75$ for group AC, and 13.54 \pm 7.99 for group $\mathrm{CP}(\mathrm{p}>0.05)$. During the follow-up, these values gradually got worse in all groups (Fig. 3). However, no significant differences were observed in Cobb angle change from the immediately postoperative to the 1-year follow-up across all groups: $-2.77 \pm 3.43,-0.95 \pm 0.57$, and $-0.98 \pm 1.04$ in groups cage, $\mathrm{AC}$, and $\mathrm{CP}(\mathrm{p}>0.05)$. The restriction of cervical ROM from the preoperative to 1- year follow-up was $-7.9 \pm 5.65$ for group cage, $-0.82 \pm 4.89$ for group $\mathrm{AC}$, and $-2.06 \pm 4.8$ for group $\mathrm{CP}(\mathrm{p}>0.05)$. The results of a multiple comparison analysis about the restriction of cervical ROM showed a significant decrease in group cage as compared to groups $\mathrm{AC}$ and $\mathrm{CP}(\mathrm{p}=0.02)$. The outcomes in group AC showed no significant differences as compared to those in group $\mathrm{CP}(\mathrm{p}>0.05)$ (Fig. 4).

\section{DISCUSSION}

Cervical degenerative diseases are defined as chronic degenerative processes associated with radiculopathy or myelopathy ${ }^{12,26}$. ACDF is a choice of surgical procedure for cervical degenerative diseases ${ }^{12}$. There are many advances in ACDF to reduce postoperative complications. However, ACDF still has problems, including cervical kyphosis, loss of disc height, and restriction of cervical ROM. The loss of cervical lordosis and disc height is deemed to be a risk factor that contributes to the progression of degenerative changes in the cervical spine, reducing the dynamic movement and increasing biomechanical stress on adjacent segments ${ }^{9,25}$. Loss of height between vertebral bodies makes narrow the foraminal space and induces secondary pain caused by nerve root compression ${ }^{28)}$.

In the present study, we evaluated the radiologic outcomes of 3 anterior fusion methods in single-level cervical disc disease, namely, stand-alone cage, PEEK-titanium combined anchored cage, and anterior cervical cage-plate. Previously, Cho

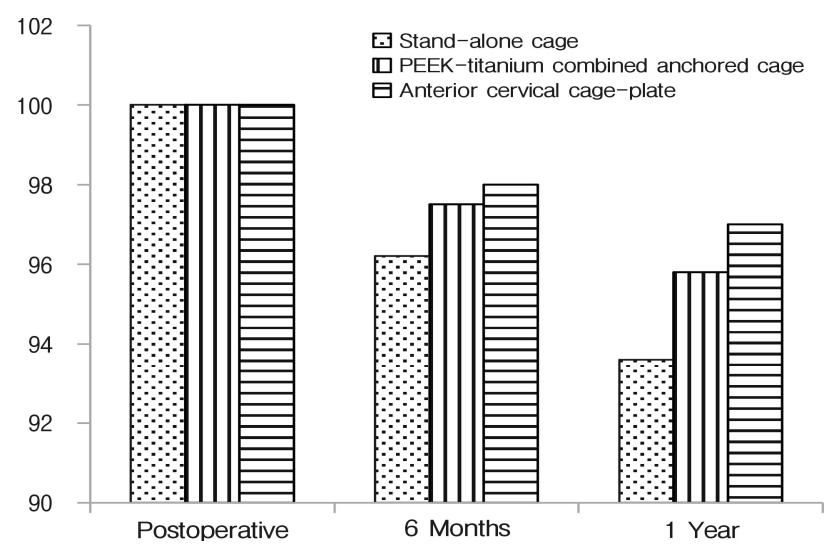

Fig. 2. To evaluate fused segment height (FSH) change of the surgical level, immediately postoperative height set to $100 \%$. While the loss of height occurring, there was not statistically si gnifi cant difference at 6 months after surgery. But, 1-year follow-up, FSH was decreased significantly compared to the postoperative image in all groups. The results showed a statistically significant decrease of FSH in group cage as compared to group $\mathrm{CP}(\mathrm{p}=0.01)$. CP, anterior cervical cage-plate.

Table 1. Preoperative characteristics

\begin{tabular}{|c|c|c|c|c|}
\hline Characteristic & Group cage $(n=20)$ & Group $\mathrm{AC}(\mathrm{n}=21)$ & Group CP $(n=26)$ & p-value \\
\hline Sex, male:female & $10: 10$ & $9: 12$ & $13: 13$ & \\
\hline Age (yr) & $52.20 \pm 10.37$ & $49.55 \pm 9.34$ & $53.75 \pm 9.77$ & 0.417 \\
\hline Mean BMD & $1.03 \pm 0.14$ & $1.05 \pm 0.18$ & $0.96 \pm 0.13$ & 0.224 \\
\hline \multicolumn{5}{|l|}{ Operation level } \\
\hline $\mathrm{C} 4-5$ & 2 & 2 & 3 & \\
\hline C5-6 & 14 & 13 & 18 & \\
\hline $\mathrm{C} 6-7$ & 4 & 6 & 5 & \\
\hline
\end{tabular}

Values are presented as number or mean \pm standard deviation.

AC, PEEK-titanium combined anchored cage; CP, anterior cervical cage-plate; BMD, bone marrow density. 
et al. ${ }^{4)}$ reported that the PEEK-titanium combined anchored cage showed a statistically better outcome in Cobb angle, disc height, subsidence rate than the stand-alone cage. Furthermore, Song et al. ${ }^{24)}$ reported that anterior cervical cage-plate is more beneficial with regard to postoperative results than the stand-alone cage. In the present study, immediate postoperative outcomes in all groups temporarily improved, but then the outcomes got worse as the time goes on. At 1-year followup, group cage had more aggravated results of the loss of FSH and cervical ROM as compared to other groups. One of factors of subsidence is the postoperative cervical micromotions ${ }^{1,8,10)}$. Stand-alone cage has a weak fixation, allowing postoperative

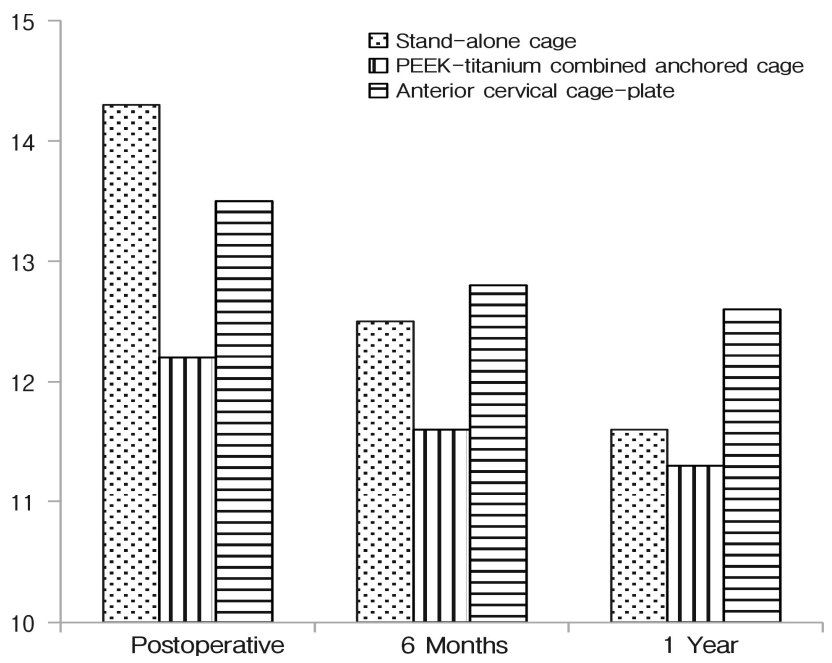

Fig. 3. The outcomes of Cobb angle worse gradually, but there was no significant difference of Cobb angle change from the immediately postoperative to the 1-year follow-up between each groups $(\mathrm{p}>0.05)$.

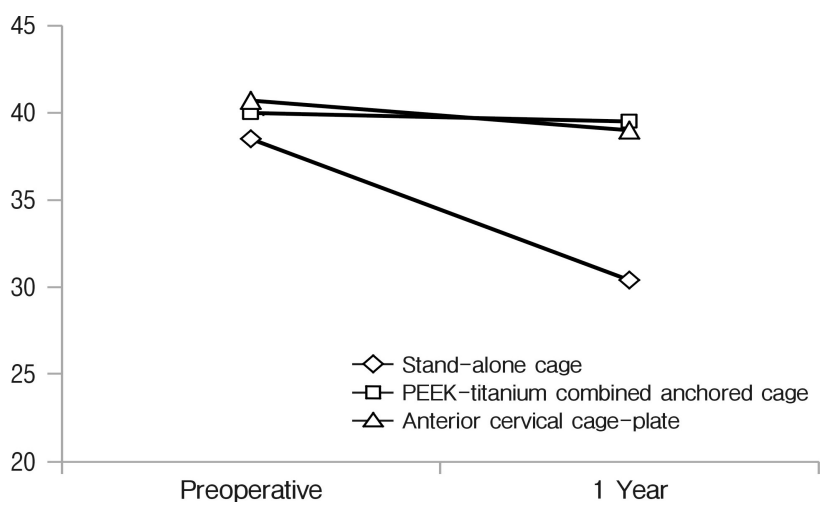

Fig. 4. Cervical range of motion (ROM) was measured as the difference of Cobb angle values between the extension and flexion positions at preoperative and 1-year follow-up. The results of the restriction of cervical ROM showed a significant decrease in group cage as compared to groups $\mathrm{AC}$ and $\mathrm{CP}(\mathrm{p}=0.02)$. $\mathrm{AC}$, PEEK-titanium combined anchored cage; $\mathrm{CP}$, anterior cervical cage-plate. micromotions to continuously occur between the surface of the cage and end plate. Subsidence has been aggravated by these continuous cervical micromotions. The ROM in patients with stand-alone cage may be limited by subsidence into the adjacent vertebrae with collapse of the intervertebral space. However, no significant difference in global cervical lordosis was observed. There could not have been enough time to aggravate the degenerative change in adjacent levels. According to Kim et al. ${ }^{11)}$, the subsidence does not affect short-term outcomes and cervical kyphosis is a long-term consequence of degenerative change. As to the comparison between groups $\mathrm{AC}$ and $\mathrm{CP}$, several previous studies reported no significant difference in radiological outcomes. The PEEK-titanium combined anchored cage is constructed of radiolucent PEEK and radio-opaque titanium alloy plate into its anterior surface. The radiolucent PEEK has a similar elastic modulus to that of human bone, which reduces the bone weakness and increases the fusion rate. But titanium aggravates fusion rates, subsidence rates versus with PEEK ${ }^{15)}$. Scholz et al. ${ }^{18)}$ reported designing the PEEK-titanium combined anchored cage using 4 different trajectory screws to limit the risk of damage to adjacent end plates and support between intervertebral bodies. This study also showed that group AC had similar radiologic outcomes as compared to group $\mathrm{CP}$. Therefore, these screws act as plate fixation to make biomechanically equivalent.

Although anterior cervical cage-plate has been reported to provide stability and reduce complications related to the use of stand-alone cage, the application of plate induced some complications, such as dysphagia, esophageal injury, and looseness of screws ${ }^{3,7,20)}$. Dysphagia is the common complication after ACDF using plate. The causes of dysphagia are adhesion and hematoma around the plate, soft tissue swelling at the operation site, thickness of the plate, and esophageal injury ${ }^{19)}$. Bazaz et al. ${ }^{3)}$ reported that the dysphagia rate increased after ACDF using plate. Using a smaller and smoother plate, Lee et al. $^{13)}$ reported a reduction in the incidence of dysphagia. To reduce complications after ACDF with anterior cervical plate, the PEEK-titanium combined anchored cage was designed for zero plate concept, as it can minimize the extent of surgery within the intervertebral disc space thereby reducing the risk of damage to vessels, adjacent soft tissues, and the esophagus, the known causes of chronic dysphagia ${ }^{13)}$. In their meta-analysis of the occurrence of dysphagia, Liu et al. ${ }^{14)}$ analyzed 266 cases of PEEK-titanium combined anchored cage and 300 cases of anterior cervical cage-plate. The authors observed that, at the final follow-up, dysphagia rate was significantly lower in the group of PEEK-titanium combined anchored cage $(0.4 \%, 1$ of 266 ) as compared to the group of anterior cervical cage-plate $(6.7 \%, 20$ of 300$)(\mathrm{p}=0.01)^{14}$. Similarly, Son et $\mathrm{al}^{23)}$ also reported that the dysphagia rate 6 months after ACDF using anterior cervical plate was $22.2 \%$ as compared with PEEK-titanium combined anchored cage $(0 \%)(\mathrm{p}=0.02)$.

The results of our study show that group AC have similar radiological outcomes as compared to group CP. Based on the data of postoperative dysphagia rate reported by several 
authors, we argue that a PEEK-titanium combined anchored cage may be a good alternative to ACDF in the patients with single-level cervical disease for reducing dysphagia.

This study has several limitations. First, this study was designed as a retrospective chart and image review. We were not able to make a more detailed comparison on each operative level, as the total number of cases was small and the follow-up periods were short. Second, the height of intraoperative distraction before the suitable cage insertion was erratic. Therefore, the results on the disc height change were estimated in percentage terms. Third, BMD may be of importance in predicting the mechanical strength of the cage-end-plate interface. In the present study, we estimated BMD based on the lumbar spine, rather than on the cervical spine. Therefore, BMD in this study typically acquired from the lumbar spine are not clearly reflected with $\mathrm{BMD}$ of the cervical spine.

\section{CONCLUSION}

In the present study, we radiologically compared stand-alone cage, PEEK-titanium combined anchored cage, and anterior cervical cage-plate with regard to incidence of complications in the treatment of single-level cervical disease. Our results suggest that, at 1-year follow-up, Groups AC and CP had better outcomes as compared to those in group cage. Furthermore, group AC had similar radiologic outcomes as in those in group $\mathrm{CP}$. Therefore, the PEEK-titanium combined anchored cage may be a good alternative procedure to reduce complications induced by plate after ACDF.

\section{CONFLCT OF INTEREST}

No potential conflict of interest relevant to this article was reported

\section{REFERENCES}

1. Barsa P, Suchomel P: Factors affecting sagittal malalignment due to cage subsidence in standalone cage assisted anterior cervical fusion. Eur Spine J 16:1395-1400, 2007

2. Bartels RH, Donk RD, Feuth T: Subsidence of stand-alone cervical carbon fiber cages. Neurosurgery 58:502-508, 2006

3. Bazaz R, Lee MJ, Yoo JU: Incidence of dysphagia after anterior cervical spine surgery: a prospective study. Spine (Phila Pa 1976) 27:2453-2458, 2002

4. Cho HJ, Hur JW, Lee JB, Han JS, Cho TH, Park JY: Cervical Stand-alone polyetheretherketone cage versus zero-profile anchored spacer in single-level anterior cervical discectomy and fusion: minimum 2-year assessment of radiographic and clinical outcome. J Korean Neurosurg Soc 58:119-124, 2015

5. Fraser JF, Härtl R: Anterior approaches to fusion of the cervical spine: a metaanalysis of fusion rates. J Neurosurg Spine 6:298303, 2007

6. Kaiser MG, Haid RW Jr, Subach BR, Barnes B, Rodts GE Jr: Anterior cervical plating enhances arthrodesis after discectomy and fusion with cortical allograft. Neurosurgery 50:229-236, 2002

7. Kalb S, Reis MT, Cowperthwaite MC, Fox DJ, Lefevre R, Theodore $\mathrm{N}$, et al: Dysphagia after anterior cervical spine surgery: incidence and risk factors. World Neurosurg 77:183-187, 2012

8. Kast E, Derakhshani S, Bothmann M, Oberle J: Subsidence after anterior cervical inter-body fusion. A randomized prospective clinical trial. Neurosurg Rev 32:207-214, 2009

9. Katsuura A, Hukuda S, Saruhashi Y, Mori K: Kyphotic malalignment after anterior cervical fusion is one of the factors promoting the degenerative process in adjacent intervertebral levels. Eur Spine J 10:320-324, 2001

10. Kettler A, Wilke HJ, Claes L: Effects of neck movements on stability and subsidence in cervical interbody fusion: an in vitro study. J Neurosurg 94(1 Suppl):97-107, 2001

11. Kim CH, Chung CK, Hahn S: Autologous iliac bone graft with anterior plating is advantageous over the stand-alone cage for seg mental lordosis in single-level cervical disc disease. Neurosurgery 72:257-265, 2013

12. Kulkarni AG, Hee HT, Wong HK: Solis cage (PEEK) for anterior cervical fusion: preliminary radiological results with emphasis on fusion and subsidence. Spine J 7:205-209, 2007

13. Lee MJ, Bazaz R, Furey CG, Yoo J: Influence of anterior cervical plate design on Dysphagia: a 2-year prospective longitudinal follow-up study. J Spinal Disord Tech 18:406-409, 2005

14. Liu W, Hu L, Wang J, Liu M, Wang X: Comparison of zero-profile anchored spacer versus plate-cage construct in treatment of cervical spondylosis with regard to clinical outcomes and incidence of major complications: a meta-analysis. Ther Clin Risk Manag 11:1437-1447, 2015

15. Niu CC, Liao JC, Chen WJ, Chen LH: Outcomes of interbody fusion cages used in 1 and 2-levels anterior cervical discectomy and fusion: titanium cages versus polyetheretherketone (PEEK) cages. J Spinal Disord Tech 23:310-316, 2010

16. Pitzen TR, Chrobok J, Stulik J, Ruffing S, Drumm J, Sova L, et al: Implant complications, fusion, loss of lordosis, and outcome after anterior cervical plating with dynamic or rigid plates: two-year results of a multi-centric, randomized, controlled study. Spine (Phila Pa 1976) 34:641-646, 2009

17. Sahjpaul RL: Esophageal perforation from anterior cervical screw migration. Surg Neurol 68:205-209, 2007

18. Scholz M, Schnake KJ, Pingel A, Hoffmann R, Kandziora F: A new zero-profile implant for stand-alone anterior cervical interbody fusion. Clin Orthop Relat Res 469:666-673, 2011

19. Shi S, Liu ZD, Li XF, Qian L, Zhong GB, Chen FJ: Comparison of plate-cage construct and stand-alone anchored spacer in the surgical treatment of three-level cervical spondylotic myelopathy: a preliminary clinical study. Spine J 15:1973-1980, 2015

20. Shin JS, Oh SH, Cho PG: Surgical outcome of a zero-profile device comparing with stand-alone cage and anterior cervical plate with iliac bone graft in the anterior cervical discectomy and fusion. Korean J Spine 11:169-177, 2014

21. Silber JS, Anderson DG, Daffner SD, Brislin BT, Leland JM, Hilibrand AS, et al: Donor site morbidity after anterior iliac crest bone harvest for single-level anterior cervical discectomy and fusion. Spine (Phila Pa 1976) 28:134-139, 2003

22. Smith GW, Robinson RA: The treatment of certain cervical-spine disorders by anterior removal of the intervertebral disc and inter- 
body fusion. J Bone Joint Surg Am 40-A:607-624, 1958

23. Son DK, Son DW, Kim HS, Sung SK, Lee SW, Song GS: Comparative study of clinical and radiological outcomes of a zeroprofile device concerning reduced postoperative Dysphagia after single level anterior cervical discectomy and fusion. J Korean Neurosurg Soc 56:103-107, 2014

24. Song KJ, Taghavi CE, Lee KB, Song JH, Eun JP: The efficacy of plate construct augmentation versus cage alone in anterior cervical fusion. Spine (Phila Pa 1976) 34:2886-2892, 2009

25. Takeshima T, Omokawa S, Takaoka T, Araki M, Ueda Y, Takakura Y: Sagittal alignment of cervical flexion and extension: lateral radiographic analysis. Spine (Phila Pa 1976) 27:E348-355, 2002
26. Tampier C, Drake JD, Callaghan JP, McGill SM: Progressive disc herniation: an investigation of the mechanism using radiologic, histochemical, and microscopic dissection techniques on a porcine model. Spine (Phila Pa 1976) 32:2869-2874, 2007

27. Thorell W, Cooper J, Hellbusch L, Leibrock L: The long-term clinical outcome of patients undergoing anterior cervical discectomy with and without intervertebral bone graft placement. Neurosurgery 43:268-273, 1998

28. Yang JJ, Yu CH, Chang BS, Yeom JS, Lee JH, Lee CK: Subsidence and nonunion after anterior cervical interbody fusion using a stand-alone polyetheretherketone (PEEK) cage. Clin Orthop Surg 3:16-23, 2011 